\title{
Hodge cohomology of invertible sheaves
}

\author{
Hélène Esnault and Arthur Ogus
}

November 28, 2018

\section{Introduction}

Let $k$ be an algebraically closed field and let $X / k$ be a smooth projective and geometrically connected $k$-scheme. Let $L$ be an invertible sheaf on $X$, and for each integer $m$, let

$$
H_{H d g}^{m}(X / k, L):=\bigoplus_{a+b=m} H^{b}\left(X, L \otimes \Omega_{X / k}^{a}\right)
$$

We wish to study how the dimensions of the $k$-vector spaces $H_{H d g}^{m}(X, L)$ and $H^{b}\left(X, L \otimes \Omega_{X / k}^{a}\right)$ vary with $L$. For example, if $k$ has characteristic zero, Green and Lazarsefeld [3] proved that for given $i, j, m$, the subloci

$$
\left\{L \in \operatorname{Pic}^{0}(X): \operatorname{dim} H^{i}\left(X, \Omega_{X}^{j} \otimes L\right) \geq m\right\}
$$

of $\operatorname{Pic}^{0}(X)$ are translates of abelian subvarieties, and Simpson [8] showed that they in fact are translates by torsions points. Both these papers use analytic methods, but Pink and Roessler [6] obtained the same results purely algebraically, using the technique of $\bmod p$ reduction and the decomposition theorem of Deligne-Illusie. A key point of their proof is the fact that

$$
\operatorname{dim} H_{H d g}^{m}(X / k, L)=\operatorname{dim} H_{H d g}^{m}\left(X / k, L^{a}\right)
$$

if $L^{n} \cong \mathcal{O}_{X}$ and $(a, n)=1([6$, Proposition 3.5]). They conjecture that equation 1 remains true in characteristic $p>0$ if $X / k$ lifts to $W_{2}$ and has dimension $\leq p$. The purpose of this note is to discuss a few aspects of this conjecture and some variants. 
Our main result (see Theorem 5) says that the conjecture is true if $n=$ $p$ and $X$ is ordinary in the sense of Bloch-Kato [1, Definition 7.2]. We also explain in section 2 some motivic variants of (1) and, in particular in Proposition 1, a proof (due to Pink and Roessler) of the characteristic zero case of (1), using the language Grothendieck Chow motives. We should remark that there are also some log versions of these questions, which we will not make explicit.

Acknowledgements: We thank D. Roessler for explaining to us his and R. Pink's analytic proof of equation 1 .

\section{A motivic variant}

Question 1 Suppose $L^{n} \cong \mathcal{O}_{X}$ for some natural number $n$. Is

$$
\operatorname{dim} H_{H d g}^{m}\left(X / k, L^{i}\right)=\operatorname{dim} H_{H d g}^{m}(X / k, L)
$$

for every $i$ relatively prime to $n$ ?

Let us explain how this question can be given a motivic interpretation. We refer to [7] for the definition of Grothendieck's Chow motives over an algebraically closed field $k$. In particular, objects are triples $(Y, p, n)$ where $Y$ is a smooth projective variety over $k, p$ is an element $C H^{\operatorname{dim}(Y)}\left(Y \times_{k} Y\right) \otimes \mathbb{Q}$, the rational Chow group of $\operatorname{dim}(Y)$-cycles, which, as a correspondence, is an idempotent, and $n$ is a natural number.

Let $\pi: Y \rightarrow X$ be a principal bundle under a group scheme $\mu$, where $X$ and $Y$ are smooth and projective over $k$. The $\mu$-torsor structure of $Y$ defines an isomorphism

$$
(\xi, y) \mapsto(y, \xi y): \mu \times_{k} Y \cong Y \times_{X} Y \subseteq Y \times_{k} Y
$$

Thus a point $\xi \in \mu(k)$ defines an irreducible closed subset $\Gamma_{\xi}$ of $Y \times_{k} Y$, the graph of the endomorphism of $Y$ defined by $\xi$. The map $\xi \mapsto \Gamma_{\xi}$ extends uniquely to a map of vector spaces

$$
\mu(k) \rightarrow C H^{\operatorname{dim}(Y)}\left(Y \times_{k} Y\right) \otimes \mathbb{Q}
$$

This map is in fact compatible with composition, and in particular if $\xi \in$ $\mathbb{Q}[\mu]$ is idempotent in the group ring $\mathbb{Q}[\mu]$, then $Y \times \xi$ is idempotent as a 
correspondence. In this case we let $Y_{\xi}$ be the Grothendieck Chow motive $(Y, \xi, 0)$.

Let $L$ be an $n$-torsion invertible sheaf on smooth projective scheme $X / k$. An $\mathcal{O}_{X}$-isomorphism $L^{n} \cong \mathcal{O}_{X}$ defines an $\mathcal{O}_{X}$-algebra structure on

$$
\mathcal{A}:=\bigoplus_{i=0}^{n-1} L^{i} .
$$

Let $\pi: Y:=\operatorname{Spec}_{X} \mathcal{A} \rightarrow X$ be the corresponding $X$-scheme. As well known, $\pi$ is a torsor under the group scheme $\mu_{n}$ of $n$th roots of unity. Indeed, locally Zariski on $X, \mathcal{A} \cong \mathcal{O}_{X}[t] /\left(t^{n}-u\right)$ for a local unit $u$, the $\mu_{n}$-action is defined by $\mathcal{A} \rightarrow \mathcal{A} \otimes \mathbb{Q}[\zeta] /\left(\zeta^{n}-1\right), t \mapsto t \zeta$, and the torsor structure is given by $\mathcal{A} \otimes \mathbb{Q}[\zeta] /\left(\zeta^{n}-1\right) \cong \mathcal{A} \otimes_{\mathcal{O}_{X}} \mathcal{A},(t, \zeta) \mapsto(t, t \zeta)$. Assuming now that $n$ invertible in $k, \mu_{n}$ is étale, hence $\pi$ is étale and $Y$ is smooth and projective over $k$. Note that the character group $X_{n}:=\operatorname{Hom}\left(\mu_{n}, \mathbf{G}_{m}\right)$ is cyclic of order $n$ with a canonical generator (namely, the inclusion $\mu_{n} \rightarrow \mathbf{G}_{m}$ ). By construction, the direct sum decomposition (2) of $\mathcal{A}$ corresponds exactly to its eigenspace decomposition according to the characters of $\mu_{n}$.

We can now apply the general construction of motives to this situation. Since $\mu_{n}$ is étale over the algebraically closed field $k$, it is completely determined by the finite group $\Gamma:=\mu_{n}(k)$, which is cyclic of order $n$. The group algebra $\mathbb{Q}[\Gamma]$ is a finite separable algebra over $\mathbb{Q}$, hence is a product of fields: $\mathbb{Q}[\Gamma]=\prod E_{e}$. There is an (indecomposable) idempotent $e$ corresponding to each of these fields, and for each $e$ we find a Chow motive $Y_{e}$.

The indecomposable idempotents of $\mathbb{Q}[\Gamma]$ can also be thought of as points of the spectrum $T$ of $\mathbb{Q}[\Gamma]$. If $K$ is a sufficiently large extension of $\mathbb{Q}$, then

$$
\begin{gathered}
T(K)=\operatorname{Hom}_{\mathrm{Alg}}(\mathbb{Q}[\Gamma], K)=\operatorname{Hom}_{\mathrm{Gr}}\left(\Gamma, K^{*}\right), \\
\text { and } K \otimes \mathbb{Q}[\Gamma] \cong K[\Gamma] \cong K^{T(K)} .
\end{gathered}
$$

Thus $T(K)$ can be identified with the character group $X_{n}$ of $\Gamma$, and is canonically isomorphic to $\mathbf{Z} / n \mathbf{Z}$, with canonical generator the inclusion $\Gamma \subseteq k$. Suppose that $K / \mathbb{Q}$ is Galois. Then $\operatorname{Gal}(K / \mathbb{Q})$ acts on $T(K)$, and the points of $T$ correspond to the $\operatorname{Gal}(K / \mathbb{Q})$-orbits. By the theory of cyclotomic extensions of $\mathbb{Q}$, this action factors through a surjective map

$$
\operatorname{Gal}(K / \mathbb{Q}) \rightarrow(\mathbf{Z} / n \mathbf{Z})^{*}
$$

and the usual action of $(\mathbf{Z} / n \mathbf{Z})^{*}$ on $\mathbf{Z} / n \mathbf{Z}$ by multiplication. Thus the orbits correspond precisely to the divisors $d$ of $n$; we shall associate to each orbit $S$ 
the index $d$ of the subgroup of $\mathbf{Z} / n \mathbf{Z}$ generated by any element of $S$. (Note that in fact the image of $d$ in $\mathbf{Z} / n \mathbf{Z}$ belongs to $S$.) We shall thus identify the indecomposable idempotents of $\mathbb{Q}[\Gamma]$ and the divisors of $n$.

Let us suppose that $k=\mathbf{C}$. Then we can consider the Betti cohomologies of $X$ and $Y$, and in particular the group algebra $\mathbb{Q}[\Gamma]$ operators on $H^{m}(Y, \mathbb{Q})$. We can thus view $H^{m}(Y, \mathbb{Q})$ as a $\mathbb{Q}[\Gamma]$-module, which corresponds to a coherent sheaf $\tilde{H}^{m}(Y, \mathbb{Q})$ on $T$. If $e$ is an idempotent of $\mathbb{Q}[\Gamma]$, then $H^{m}\left(Y_{e}, \mathbb{Q}\right)$ is the image of the action of $e$ on $H^{m}(Y, \mathbb{Q})$, or equivalently, it is the stalk of the sheaf $\tilde{H}^{m}(Y, \mathbb{Q})$ at the point of $T$ corresponding to $e$, or equivalently, it is $H^{m}(Y, \mathbb{Q}) \otimes E_{e}$ where the tensor product is taken over $\mathbb{Q}[\Gamma]$. If $K$ is a sufficiently large field as above, then equation (4) induces an isomorphism of $K$-vectors spaces:

$$
H^{m}\left(Y_{e}, \mathbb{Q}\right) \otimes_{\mathbb{Q}} K \cong \bigoplus\left\{H^{m}(Y, K)_{t}: t \in T^{e}(K)\right\},
$$

where here $T^{e}(K)$ means the set of points of $T(K)$ in the Galois orbit corresponding to $e$, and $H^{m}(Y, K)_{t}$ means the $t$-eigenspace of the action of $\Gamma$ on $H^{m}(Y, \mathbb{Q}) \otimes_{\mathbb{Q}} K$. The de Rham and Hodge cohomologies of $Y_{e}$ are defined in the same way: they are the images of the actions of the idempotent $e$ acting on the $k$-vector spaces $H_{D R}(Y / k)$ and $H_{H d g}(Y / k)$.

The following result is due to Pink and Roessler. Their article [6] contains a proof using reduction modulo $p$ techniques and the results of [2]; the following analytic argument is based on oral communications with them.

Proposition 1 The answer to question 1 is affirmative if $k$ is a field of characteristic zero.

Proof: As both sides of the equality in Question 1 satisfy base change with respect to field extensions, we may assume that $k=\mathbf{C}$. Let $i \rightarrow t_{i}$ denote the isomorphism $\mathbf{Z} / n \mathbf{Z} \cong T(\mathbf{C})$. For each divisor $e$ of $n$ there is a corresponding idempotent $e$ of $\mathbb{Q}[\Gamma] \subseteq K[\Gamma]$, the sum over all $i$ such that $t_{i} \in T^{e}(\mathbf{C})$. Consider the Hodge cohomology of the motive $Y_{e}$ :

$$
\begin{aligned}
H_{H d g}^{m}\left(Y_{e} / \mathbf{C}\right):=H_{H d g}^{m}(Y / \mathbf{C}) \otimes_{\mathbb{Q}[\Gamma]} E_{e} & \cong H_{H d g}^{m}(Y / \mathbf{C}) \otimes_{\mathbf{C}[\Gamma]}\left(\mathbf{C} \otimes E_{e}\right) . \\
& \cong \bigoplus\left\{H_{H d g}^{m}(Y / \mathbf{C})_{i}: i \in T^{e}(k)\right\} .
\end{aligned}
$$

Since $\pi: Y \rightarrow X$ is finite and étale,

$$
\begin{aligned}
H^{b}\left(Y, \Omega_{Y / \mathbf{C}}^{a}\right) \cong H^{b}\left(X, \pi_{*} \pi^{*} \Omega_{X / \mathbf{C}}^{a}\right) & \cong H^{b}\left(X, \Omega_{X / \mathbf{C}}^{a} \otimes \pi_{*} \mathcal{O}_{Y}\right) \\
& \cong \bigoplus\left\{H^{b}\left(X, \Omega_{X / \mathbf{C}}^{a} \otimes L^{i}\right): i \in \mathbf{Z} / n \mathbf{Z}\right\}
\end{aligned}
$$


Thus

$$
H_{H d g}^{m}(Y / \mathbf{C}) \cong \bigoplus\left\{H_{H d g}^{m}\left(X, L^{i}\right): i \in \mathbf{Z} / n \mathbf{Z}\right\}
$$

and hence from the explicit description of the action of $\mu_{n}$ on $\mathcal{A}$ above it follows that

$$
H_{H d g}^{m}\left(Y_{e} / \mathbf{C}\right)=\bigoplus\left\{H_{H d g}^{m}\left(X, L^{i}\right): i \in T_{e}(\mathbf{C})\right\}
$$

The Hodge decomposition theorem for $Y$ provides us with an isomorphism:

$$
H_{H d g}^{m}(Y / \mathbf{C}) \cong \mathbf{C} \otimes H^{m}(Y, \mathbb{Q})
$$

compatible with the action of $\mathbb{Q}[\Gamma]$. This gives us, for each idempotent $e$, an isomorphism of $\mathbf{C} \otimes E_{e}$-modules.

$$
H_{H d g}^{m}\left(Y_{e} / \mathbf{C}\right) \cong \mathbf{C} \otimes H^{m}\left(Y_{e}, \mathbb{Q}\right)
$$

The action on $\mathbf{C} \otimes H^{m}\left(Y_{e}, \mathbb{Q}\right)$ on the right just comes from the action of $E_{e}$ on $H^{m}\left(Y_{e}, \mathbb{Q}\right)$ by extension of scalars. Since $E_{e}$ is a field, $H^{m}\left(Y_{e}, \mathbb{Q}\right)$ is free as an $E_{e}$-module, and hence the $\mathbf{C} \otimes E_{e}$-module $H_{H d g}^{m}\left(Y_{e} / \mathbf{C}\right)$ is also free. It follows that its rank is the same at all the points $t \in T^{e}(\mathbf{C})$, affirming Question 1.

Let us now formulate a motivic analog of Question 1 in characteristic $p$.

Question 2 Suppose that $k$ is an algebraically closed field of characteristic $p$ and $(n, p)=1$. Let $\ell$ be a prime different from $p$, let $e$ be a divisor of $n$, and let $E_{e}$ be the corresponding factor of $\mathbb{Q}[\Gamma]$. Is it true that each $H^{m}\left(Y_{e}, \mathbb{Q}_{\ell}\right)$ is a free $\mathbb{Q}_{\ell} \otimes E_{e}$-module? And is it true that $H_{c r i s}^{m}\left(Y_{e} / W\right) \otimes \mathbb{Q}$ is a free $W \otimes E_{e}$-module, where $W:=W(k)$ ?

If $K$ is an extension of $\mathbb{Q}_{\ell}$ which contains a primitive $n$th root of unity, then as above we have an eigenspace decomposition:

$$
K \otimes H^{m}\left(Y, \mathbb{Q}_{\ell}\right) \cong \bigoplus\left\{H^{m}(Y, K)_{t}: t \in T(K)\right\}
$$

and this question asks whether the $K$-dimension of the $t$-eigenspace is constant over the orbits $T_{e}(K) \subseteq T(K)$.

Suppose first that $X / k$ lifts to characteristic zero, i.e., that there exists a complete discrete valuation ring $V$ with residue field $k$ and fraction field of characteristic zero and a smooth proper $\tilde{X} / V$ whose special fiber is $X / k$. 
Then we claim that since $L$ is $n$-torsion, it lifts uniquely to an $n$-torsion sheaf on $\tilde{X}$. By Grothendieck's existence theorem, it is enough to check that $L$ lifts to the formal completion $\mathcal{X}$ of $X$ along $X$, and hence it is enough to check that the lifting can be done uniquely step by step. Let $X_{m}$ be the closed subscheme of $\mathcal{X}$ defined by $\pi^{m+1}$, where $\pi$ is a uniformizing parameter of $V$. Note that since $X / k$ is smooth and geometrically connected, $k \cong H^{0}\left(X, \mathcal{O}_{X}\right)$, so the map $H^{0}\left(\mathcal{X}, \mathcal{O}_{\mathcal{X}}\right) \rightarrow H^{0}\left(X, \mathcal{O}_{X}\right)$ is surjective, $H^{1}\left(\mathcal{X}, \mathcal{O}_{\mathcal{X}}\right)$ is torsion free, and $V / \pi^{m=1} V \cong H^{0}\left(X_{m}, \mathcal{O}_{X_{m}}\right)$ for all $m$. We have an exact sequence:

$$
0 \rightarrow \mathcal{O}_{X} \rightarrow \mathcal{O}_{X_{m}}^{*} \rightarrow \mathcal{O}_{X_{m-1}}^{*} \rightarrow 0,
$$

where the first map is induced by $a \mapsto 1+\pi^{m} a$. This gives an exact sequence

$$
H^{1}\left(X, \mathcal{O}_{X}\right) \stackrel{a}{\longrightarrow} H^{1}\left(\mathcal{X}_{m}, \mathcal{O}_{X_{m}}^{*}\right) \stackrel{r}{\longrightarrow} H^{1}\left(X_{m-1}, \mathcal{O}_{X_{m-1}}^{*}\right) \stackrel{c}{\longrightarrow} H^{2}\left(X, \mathcal{O}_{X}\right) ;
$$

the map $a$ is injective because the map $V^{*} \rightarrow H^{0}\left(X_{m-1}, \mathcal{O}_{X_{m-1}}^{*}\right)$ is surjective. Since multiplication by $n$ is bijective on $H^{i}\left(X, \mathcal{O}_{X}\right)$, the map $r$ in the sequence above induces a bijection on the $n$-torsion subgroups. Indeed if $\eta$ is an element of $H^{1}\left(X_{m-1}, \mathcal{O}_{X_{m-1}}^{*}\right)$ and $n \eta=0$, then $n c(\eta)=0$, hence $c(\eta)=0$ and $\eta=r(\zeta)$ for some $\zeta$. Since $r(n \zeta)=0$, there exists a unique $\xi$ with $a(\xi)=n \zeta$, and there is then a unique $\xi^{\prime}$ with $\xi=n \xi^{\prime}$. Then $\zeta^{\prime}:=\zeta-a\left(\xi^{\prime}\right)$ is the unique $n$-torsion lifting of $\eta$. Hence $Y:=\operatorname{Spec}_{X} \oplus L^{i}$ also lifts to $V$, as well as the action of the group-scheme $\mu_{n}$. Then by the étale to Betti comparison theorems, we see that the answer to Question 2 is affirmative.

In fact, the lifting hypothesis is superfluous, but this takes a bit more work.

Claim 2 The answer to Question Q is affirmative.

Proof: This is clear if $\mathbb{Q}_{\ell} \otimes E_{e}$ is a field, by the same argument as in characteristic zero. If $(\ell, n)=1$, this is the case if and only if $(\mathbf{Z} / e \mathbf{Z})^{*}$ is cyclic and generated by $\ell$. More generally, there is a decomposition into a product of fields $\mathbb{Q}_{\ell}[\Gamma] \cong \prod E_{\ell, e}$, where now $e$ ranges over the orbits of $\mathbf{Z} / n \mathbf{Z}$ under the action of the cyclic subgroup of $(\mathbf{Z} / n \mathbf{Z})^{*}$ generated by $\ell$ (assuming $\ell$ is relatively prime to $n$ ). This shows that at least that the dimension of $H^{m}(Y, K)_{t}$ is, as a function of $t$, constant over the $\ell$-orbits.

Let $K$ be an algebraically closed field containing $\mathbb{Q}_{\ell}$ for all primes $\ell \neq p$, and containing $W(k)$. For $\ell \neq p$ let $V_{\ell}:=H^{m}\left(Y_{e ́ t}, \mathbb{Q}_{\ell}\right) \otimes_{\mathbb{Q} \ell} K$, and let $V_{p}:=$ $H^{m}\left(Y_{\text {cris }}, W(k)\right) \otimes_{W(k)} K$. Then each $V_{\ell}$ is a finite-dimensional representation 
of $\Gamma$, and the isomorphism (5) is just its decomposition as a direct sum of irreducible representations:

$$
V_{\ell} \cong \bigoplus\left\{n_{\ell, i} V_{i}: i \in \mathbf{Z} / n \mathbf{Z}\right\}
$$

where $V_{i}=K$, with $\gamma \in \Gamma$ acting by multiplication by $\gamma^{i}$. By [4, Theorem 2.2)] (and [5] for the existence of cycle class in crystalline cohomology) the trace of any $\gamma \in \Gamma$ acting on $V_{\ell}$ is independent of $\ell$, including $\ell=p$. Since $\Gamma$ is a finite group, it follows from the independence of characters that for each $i, n_{i}:=n_{\ell, i}$ is independent of $\ell$. We saw above that $n_{\ell, \ell i}=n_{\ell, i}$ if $(\ell, n)=1$ and $\ell \neq p$, so that in fact $n_{\ell i}=n_{i}$ for all $\ell \neq p$ with $(\ell, n)=1$. Since the group $(\mathbf{Z} / n \mathbf{Z})^{*}$ is generated by all such $\ell$, it follows that $n_{i}$ is indeed constant over the orbits.

What does this tell us about Question 1? If $(p, n)=1$ and $k$ is algebraically closed, $W[\Gamma]$ is still semisimple, and can be written canonically as a product of copies of $W$, indexed by $i \in T(W) \cong \mathbf{Z} / n \mathbf{Z}$. For every $t \in T(W) \cong T(k)$, we have an injective base change map from crystalline to de Rham cohomology: $k \otimes H^{m}(Y / W)_{t} \rightarrow H^{m}(Y / k)_{t}$.

Question 3 In the above situation, is $H^{q}(Y / W)$ torsion free when $(p, n)=$ 1 ?

If the answer is yes, then the maps $k \otimes H^{m}(Y / W)_{t} \rightarrow H^{m}(Y / k)_{t}$ are isomorphisms, and this means that we can compute the dimensions of the de Rham eigenspaces from the $\ell$-adic ones. Assuming also that the Hodge to de Rham spectral sequence of $Y / k$ degenerates, this should give an affirmative answer to Question 1. Note that if $X / k$ lifts $\bmod p^{2}$, the same is true of $Y / k$, and if the dimension is less or equal to $p$, the latter is true by [2].

Of course, there is no reason for Question 3 to have an affirmative answer in general. Is there a reasonable hypothesis on $X$ which guarantees it? For example, is it true if the crystalline cohomology of $X / W$ is torsion free?

\section{The $p$-torsion case in characteristic $p$}

Let us assume from now on that $k$ has characteristic $p$. In this case we can reduce question 11 to a question about connections, using the following construction of [2]. 
Proposition 3 Let $L$ be an invertible sheaf on a smooth proper variety $X$ defined over a perfect field $k$ and let $\nabla$ be the Frobenius descent connection on $L^{p}$. Suppose that $X / k$ lifts to $W_{2}$ and has dimension at most $p$. Then for every natural number $m$,

$$
h_{D R}^{m}\left(X / k,\left(L^{p}, \nabla\right)\right)=h_{H d g}^{m}(X / k, L)
$$

In particular, if $L^{p} \cong \mathcal{O}_{X}$ and $\omega:=\nabla(1)$, then for any integer a,

$$
h_{H d g}^{m}\left(X / k, L^{a}\right)=h_{D R}^{m}\left(X / k,\left(\mathcal{O}_{X}, d+a \omega\right)\right) .
$$

If $p$ divides a, this just means the degeneration of the Hodge to de Rham spectral sequence for $\left(\mathcal{O}_{X}, d\right)$.

Proof: Let $F: X \rightarrow X^{\prime}$ be the relative Frobenius map, let $\pi: X^{\prime} \rightarrow X$ be the base change map, and and let $H d g_{X^{\prime} / k}^{\cdot}$ denote the Hodge complex of $X^{\prime} / k$, i.e., the direct sum $\oplus_{i} \Omega_{X^{\prime} / k}^{i}[-i]$. Recall from [2] that the lifting yields an isomorphism in the bounded derived category of $\mathcal{O}_{X^{\prime}}$-modules:

$$
H d \dot{g}_{X^{\prime} / k} \cong F_{*}\left(\Omega_{X / k}^{\cdot}, d\right)
$$

Tensoring this isomorphism with $L^{\prime}:=\pi^{*} L$ and using the projection formula for $F$, we find an isomorphism

$$
H d{\dot{g_{X^{\prime} / k}}} \otimes L^{\prime} \cong F_{*}\left(\Omega_{X / k}^{\cdot} \otimes L^{p}, \nabla\right)
$$

Hence

$$
H_{H d g}^{m}(X / k, L) \stackrel{F_{k}^{*} \cong}{\longrightarrow} H_{H d g}^{m}\left(X^{\prime} / k, L^{\prime}\right) \stackrel{F_{*} \cong}{\longleftarrow} H_{D R}^{m}\left(X,\left(L^{p}, \nabla\right)\right) .
$$

If $L^{p}=\mathcal{O}_{X}$, the corresponding Frobenius descent connection $\nabla$ on $\mathcal{O}_{X}$ is determined by $\omega_{L}:=\nabla(1)$. It follows from the tensor product rule for connections that $\omega_{L^{a}}=a \omega_{L}$ for any integer $a$.

This suggests the following:

Question 4 Let $\omega$ be a closed one-form on $X$ and let $c$ be a unit of $k$. Is the dimension of $H_{D R}^{m}\left(X,\left(\mathcal{O}_{X}, d+c \omega\right)\right)$ independent of $c$ ? 
Remark 4 Some properness is necessary, since the $p$-curvature of $\nabla_{\omega}:=$ $d+\omega$ can change from zero to non-zero as one multiplies by an invertible constant. If the $p$-curvature is non-zero, then the sheaf $\mathcal{H}^{0}\left(\Omega_{X / k}^{\circ}, d_{\omega}\right)$ vanishes, and hence so does $H^{0}\left(X, \Omega_{X / k}^{*}, d_{\omega}\right)$. If the $p$-curvature vanishes, then $\mathcal{H}^{0}\left(\Omega_{X / k}, d_{\omega}\right)$ is an invertible sheaf $L$, which can have nontrivial sections if $X$ is allowed to shrink.

We can answer Question 4 under a strong hypothesis.

Theorem 5 Suppose that $X / k$ is smooth, proper, and ordinary in sense of Bloch and Kato [1, Definition 7.2]: $H^{i}\left(X, B_{X / k}^{j}\right)=0$ for all $i, j$, where

$$
B_{X / k}^{j}:=\operatorname{Im}\left(d: \Omega_{X / k}^{j-1} \rightarrow \Omega_{X / k}^{j}\right)
$$

Then the answer to question 4 is affirmative. Hence if $X / k$ lifts to $W_{2}$, has dimension at most $p$, and if $n=p$, the answer to Question 1 is also affirmative.

We begin with the following lemmas.

Lemma 6 Let $\omega$ be a closed one-form on $X$, and let

$$
d_{\omega}:=d+\omega \wedge \quad: \quad \Omega_{X / k}^{\cdot} \rightarrow \Omega_{X / k}^{\cdot+1} .
$$

Then the standard exterior derivative induces a morphism of complexes:

$$
\left(\Omega_{X / k}^{\cdot}, d_{\omega}\right) \stackrel{d}{\longrightarrow}\left(\Omega_{X / k}^{\cdot}, d_{\omega}\right)[1] .
$$

Proof: If $\alpha$ is a section of $\Omega_{X / k}^{q}$,

$$
\begin{aligned}
d d_{\omega}(\alpha) & =d(d \alpha+\omega \wedge \alpha) \\
& =d d \alpha+d \omega \wedge \alpha-\omega \wedge d \alpha \\
& =-\omega \wedge d \alpha
\end{aligned}
$$

Since the sign of the differential of the complex $\left(\Omega_{X / k}, d_{\omega}\right)[1]$ is the negative of the sign of the differential of $\left(\Omega_{X / k}^{*}, d_{\omega}\right)$,

$$
\begin{aligned}
d_{\omega} d(\alpha) & =-(d+\omega \wedge)(d \alpha) \\
& =-\omega \wedge d \alpha
\end{aligned}
$$


Lemma 7 Let $Z^{\cdot}:=\operatorname{ker}(d) \subseteq\left(\Omega_{X / k}^{\cdot}, d_{\omega}\right)$ and $B^{\cdot}:=\operatorname{Im}(d)[-1] \subseteq\left(\Omega_{X / k}^{\cdot}, d_{\omega}\right)$. Then for any $a \in k^{*}$, there are natural isomorphisms

$$
\begin{gathered}
\left(Z^{*}, d_{\omega}\right) \stackrel{\lambda_{a}}{\longrightarrow}\left(Z^{*}, d_{a \omega}\right) \\
\left(B^{\cdot}, d_{\omega}\right) \stackrel{\lambda_{a}}{\longrightarrow}\left(B^{*}, d_{a \omega}\right)
\end{gathered}
$$

Proof: It is clear that the boundary map $d_{\omega}$ on $Z \cdot$ and on $B^{*}$ is just wedge product with $\omega$. Then $\lambda_{a}$ is defined to be multiplication by $a^{i}$ in degree $i$, for example:

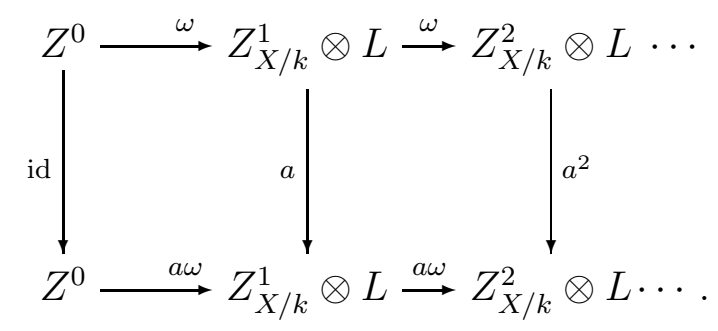

Proof of Theorem 5 We have an exact sequence:

$$
0 \rightarrow\left(Z^{\cdot}, d_{\omega}\right) \rightarrow\left(\Omega_{X / k}^{\cdot}, d_{\omega}\right) \rightarrow\left(B^{*}, d_{\omega}\right)[1] \rightarrow 0
$$

Now suppose that $X / k$ is ordinary. Then the $E_{1}$ term of the first spectral sequence for $\left(B^{*}, d_{\omega}\right)$ is $E_{1}^{i, j}=H^{j}\left(X, B^{i}\right)=0$, and it follows that the hypercohomology of $\left(B^{*}, d_{\omega}\right)$ vanishes, for every $\omega$. Hence the natural map $H^{q}\left(Z^{*}, d_{\omega}\right) \rightarrow H^{q}\left(\Omega_{X / k}^{\cdot}, d_{\omega}\right)$ is an isomorphism. Since the dimension of $H^{q}\left(Z^{*}, d_{\omega}\right)$ is unchanged when $\omega$ is multiplied by a unit of $k$, the same is true of $H^{q}\left(\Omega_{X / k}^{*}, d_{\omega}\right)$. This completes the proof of Theorem 5 .

Remark 8 A simple Riemann-Roch computation shows that on curves, question 1 always has a positive answer. However, our theorem has two extra-assumptions, namely the torsion order should precisely be $p$ and the variety should be ordinary. We are unable to determine if our additional assumptions are superfluous.

Remark 9 In the absence of the ordinarity hypothesis, one can ask if the rank of the boundary map

$$
\partial_{\omega}: H^{q}\left(B^{\cdot}, d_{\omega}[1]\right) \rightarrow H^{q+1}\left(Z^{\cdot}, d_{\omega}\right)
$$


changes if $\omega$ is multiplied by a unit of $k$. To analyze this question, let

$$
c_{\omega}:\left(B^{*}, \omega\right) \rightarrow\left(Z^{*}, \omega\right)
$$

be the morphism in the derived category defined by the exact sequence 6, so that $\partial_{\omega}$ can be identified with $H^{q-1}\left(c_{\omega}\right)$. Similarly, the exact sequence

$$
0 \rightarrow\left(Z^{*}, \omega\right) \rightarrow\left(\Omega^{*}, \omega\right) \rightarrow\left(B^{*}, \omega\right)[1] \rightarrow 0
$$

defines a morphism

$$
a_{\omega}:\left(B^{*}, \omega\right) \rightarrow\left(Z^{*}, \omega\right) .
$$

There is also an inclusion morphism:

$$
b_{\omega}:\left(B^{*}, \omega\right) \rightarrow\left(Z^{*}, \omega\right) .
$$

Then it is not difficult to check that $c_{\omega}=a_{\omega}+b_{\omega}$. If $a \in k^{*}$, we have isomorphisms of complexes

$$
\begin{aligned}
& \lambda_{a}:\left(Z^{*}, \omega\right) \rightarrow\left(Z^{*}, a \omega\right) \\
& \lambda_{a}:\left(B^{*}, \omega\right) \rightarrow\left(B^{*}, a \omega\right)
\end{aligned}
$$

Using these as identifications, one can check that $c_{a \omega}=a^{-1} a_{\omega}+b_{\omega}$. This would suggest a negative answer to Question 4 , but we do not have an example.

\section{References}

[1] Bloch, S.; Kato, K.: p-adic étale cohomology, Inst. Hautes Études Sci. Publ. Math. No. 63 (1986).

[2] Deligne, P.; Illusie, L.: Relèvements modulo $p^{2}$ et décomposition du complexe de de Rham, Invent. Math. 89 (1987), no. 2, 247-270.

[3] Green, M.; Lazarsfeld, R.: Higher obstructions to deforming cohomology groups of line bundles, J. Am. Math. Soc. 4 (1991), no 1, 87-103.

[4] Katz, N.; Messing, W.: Some consequences of the Riemann hypothesis for varieties over finite fields, Invent. Math. 23 (1974), 73-77. 
[5] Petrequin, D.: Classes de Chern et classes de cycles en cohomologie rigide, Bull. Soc. Math. France 131 (2003), no 1, 59-121.

[6] Pink, R.; Roessler, D.: A conjecture of Beauville and Catanese revisited, Math.Ann. 330 (2004), no 2, 293-308.

[7] Scholl, A.: Classical motives. Motives (Seattle, WA, 1991), 163-187, Proc. Sympos. Pure Math., 55, Part 1, Amer. Math. Soc., Providence, RI, 1994.

[8] Simpson, C.: Subspaces of moduli spaces of rank one local systems, Ann. Sc. Éc. Norm. Sup. (4) 26 (1993), no 3, 361-401. 